Melatonin in perimenopausal and postmenopausal women : associations with mood, sleep, climacteric symptoms, and quality of life

\title{
Toffol, Elena
}

2014-05

Toffol , E , Kalleinen , N , Haukka , J , Vakkuri , O , Partonen , T \& Polo-Kantola , P 2014 , ' Melatonin in perimenopausal and postmenopausal women : associations with mood, sleep, climacteric symptoms, and quality of life ' , Menopause , vol. 21 , no. 5 , pp. 493-500 . https://doi.org/10.1097/GME.0

http://hdl.handle.net/10138/301136

https://doi.org/10.1097/GME.0b013e3182a6c8f3

cc_by

acceptedVersion

Downloaded from Helda, University of Helsinki institutional repository.

This is an electronic reprint of the original article.

This reprint may differ from the original in pagination and typographic detail.

Please cite the original version. 
Melatonin in perimenopausal and postmenopausal women: associations with mood,

2 sleep, climacteric symptoms and quality of life

3 Running Title: Melatonin in perimenopause and postmenopause

4

6

7

8

${ }^{1}$ Department of Mental Health and Substance Abuse Services, National Institute for Health and Welfare THL, Helsinki, Finland

${ }^{2}$ Department of Psychiatry, University of Helsinki, Helsinki, Finland

${ }^{3}$ Sleep Research Unit, Department of Physiology, University of Turku, Turku, Finland ${ }^{4}$ Heart Centre, Turku University Hospital, Turku, Finland

${ }^{5}$ Department of Public Health, Hjelt Institute, University of Helsinki, Helsinki, Finland ${ }^{6}$ Institute of Biomedicine, University of Oulu, Oulu, Finland

${ }^{7}$ Department of Obstetrics and Gynecology, Turku University Hospital, Turku, Finland

\section{列} .

Financial disclosure: The study was financially supported by a European Commission Grant (QLK6-CT-200000499), by the Väinö and Laina Kivi Foundation, The Finnish Menopause Society Foundation, The Finnish Medical Foundation and The Turku University Foundation (PP-K). Further financial support was provided by grants from the Research Foundation of the University of Helsinki, from the Center for International Mobility, from the Lundbeck Foundation, and by the National Graduate School of Clinical Investigation (Helsinki, Finland) (ET). The authors declare that they have no conflicts of interest. Institute for Health and Welfare (THL), Mannerheimintie 170, P.O. Box 30, FI-00271 Helsinki, Finland. Tel.: +358 295248736. E-mail: elena.toffol@thl.fi.

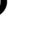


31 Objective: Melatonin synthesis and secretion are partly modulated by estrogen and progesterone. Changes in melatonin concentrations, possibly related to the menopausal

33 transition, may be associated with climacteric mood, sleep and vasomotor symptoms. The 34 aims of this study were to compare the serum concentrations of melatonin in perimenopausal and postmenopausal women, and to evaluate its influence on mood, sleep, vasomotor symptoms and quality of life. Methods: We analyzed data of 17 perimenopausal (43-51 years) and 18 postmenopausal (58-71 years) healthy women who participated in a prospective study. During the study night (21:00-09:00 hr) serum melatonin was sampled at 20-minute (21:00-24:00 hr; 06:00-09:00 hr) and one-hour (24:00-06:00 hr) intervals. Questionnaires were used to assess depression (Beck Depression Inventory, BDI), anxiety (State-Trait

41 Anxiety Inventory, STAI), insomnia and sleepiness (Basic Nordic Sleep Questionnaire, BNSQ), subjective sleep quality, vasomotor symptoms, and the quality of life (EuroQoL).

43 Results: Postmenopausal women had lower nighttime serum melatonin concentrations than

44 perimenopausal women. The duration of melatonin secretion tended to be shorter in 45 postmenopause, while the melatonin peak time did not differ. Mean melatonin concentrations 46 and exposure levels did not correlate with FSH or E2, BMI, BDI, STAI, BNSQ insomnia, 47 BNSQ sleepiness, subjective sleep, climacteric vasomotor score or the quality of life. In 48 perimenopause, the later the melatonin peak, the higher the level of anxiety $(p=0.022)$, and the 49 longer the melatonin secretion, the better the quality of life $(p<0.001)$. Conclusions:

50 Longitudinal research is needed to better understand the possible contributive role of 51 menopause on lower melatonin levels.

54 melatonin, perimenopause, postmenopause, mood, sleep, quality of life. 
57 Melatonin is a hormone produced primarily by the pineal gland and by the retina, skin and 58 gastrointestinal tract. ${ }^{1}$ The synthesis and secretion of melatonin follow a circadian rhythm and 59 are indirectly regulated by the light/dark cycle, with light having an inhibitory and darkness a 60 stimulatory effect. ${ }^{2}$ According to animal and human studies, estrogen and, at a less extent, 61 progesterone ${ }^{3-6}$ also contribute to direct and indirect modulation of melatonin synthesis and 62 secretion. In addition, melatonin concentration is influenced by administration of 63 gonadotropin-releasing hormone agonists, ${ }^{7,8}$ and in general, the circulating melatonin 64 concentrations seem to be high when endogenous estrogen levels are low, like in women 65 using oral contraception..$^{9}$ Reciprocally, it has been suggested that melatonin has a 66 regulatory, ${ }^{10}$ mostly inhibitory, effect on reproduction, probably by down-regulating the 67 hypothalamic-pituitary-ovarian axis and modulating estrogen synthesis in peripheral tissues, ${ }^{11}$ 68 such as in the breast, muscles and adipose tissue. For instance, Voordouw et al. ${ }^{12}$ found a 69 reduced ovarian function, decreased luteinizing hormone and estrogen and progesterone levels 70 after 4-month melatonin (or melatonin-progestin combinations) administration in adult 71 women. Elevated levels of plasma/serum nocturnal melatonin (concentration, area under 72 curve AUC, peak amplitude) have also been associated with reproductive malfunctions ${ }^{13}$ such 73 as amenorrhea in women ${ }^{7,14}$ and hypogonadism in men. ${ }^{15}$

74 Although the peak serum melatonin concentrations, as well as the total amount of urinary 75 melatonin, decrease physiologically with age both in men and women, ${ }^{16,17}$ probably due to 76 pineal aging, it is possible that in women the mean nocturnal melatonin concentrations, as 77 well as the melatonin peak time, the amplitude and duration of melatonin secretion vary during the menopausal transition in relation to changes in gonadal hormone levels. However, the literature on this issue is sparse and inconsistent. ${ }^{18-20}$ 
80 Because of this possible reciprocal relationship between melatonin and gonadal hormones,

81 and since long-term administration of melatonin was found to partly improve the quality of

82 life (with regard to the physical domain) in perimenopausal women, ${ }^{21}$ it is possible to

83 hypothesize that melatonin may also contribute to mitigate other symptoms associated with

84 the perimenopausal hormonal fluctuations. Among others, depressive and anxiety symptoms

85 and disorders are common during the perimenopause. ${ }^{22-24}$ Impaired sleep quality is also

86 common during the menopausal transition and in postmenopause. ${ }^{25-29}$ Melatonin is known to

87 influence mood and sleep. In fact, even with some inconsistencies, melatonin secretion seems

88 to be lower in individuals having depression,,$^{30-35}$ and melatonin or melatonin receptor

89 agonists have antidepressants, anxiolytic and sleep-promoting effects. ${ }^{36-39}$ It is therefore

90 plausible that changes in melatonin concentrations related to both aging and transition to

91 menopause may at least partly explain depressive, anxiety and sleep symptoms as well as

92 climacteric vasomotor symptoms, typically experienced by women during perimenopause and

93 postmenopause.

94 Given the general lack of data on the association between melatonin concentration and

95 secretion pattern and the transition to menopause, the aim of this work was to describe the

96 melatonin levels in perimenopausal and postmenopausal women. We hypothesized that

97 postmenopausal women have lower mean nighttime serum melatonin concentrations, as well

98 as lower melatonin exposure levels and shorter duration of nighttime secretion than

99 perimenopausal women. Our further aim was to evaluate the relationship between melatonin

100 levels and depressive, anxiety, sleep and vasomotor symptoms, as well as quality of life in

101 perimenopausal and postmenopausal women. We hypothesized that low melatonin

102 concentrations and exposure levels, and shorter duration of secretion associate with more

103 symptoms, especially in postmenopausal women. 
106 The current study was part of a larger survey evaluating the effects of menopause on sleep and 107 cognition. The women were recruited through advertisements in the local newspapers in the area 108 of Turku, Finland; 17 of them were perimenopausal (aged 43-51 years), and 18 postmenopausal 109 (aged 58-71 years). Perimenopausal status was defined by the serum follicle stimulating hormone 110 (FSH) level $(<23 \mathrm{IU} / \mathrm{mL})$ and an ongoing regular or irregular menstrual cycle. Postmenopause 111 was determined by age ( $\geq 58$ years) and at least 12 months of amenorrhea.

112 The exclusion criteria included presence of a mental, cardiovascular (with the exception of 113 drug-treated balanced hypertension), endocrine (with the exception of drug-treated balanced 114 hyperlipidemia), pulmonary, neurological or specific sleep disorder; malignancies; alcohol 115 abuse, smoking, excessive caffeine intake ( $>5$ cups per day) and use of other substances that 116 are known to affect the central nervous system. In addition, women suffering from other 117 conditions possibly affecting sleep (e.g. fibromyalgia and anemia) were excluded. All women 118 had normal levels of blood hemoglobin, leukocytes, thrombocytes and serum thyrotropin. One 119 perimenopausal woman and 13 postmenopausal women had previously used hormone therapy 120 (HT), and a washout period of at least 12 months was required. More details about the data 121 collection and study design have already been described elsewhere. ${ }^{40}$ After receiving oral and 122 written information, all participants gave written informed consent. The study was approved 123 by the Ethics Committees of Turku University Hospital and of University of Turku, Finland. 124 The participants kept a sleep diary during the three weeks before and one week after the study 125 to verify their sleep-wake rhythms. All women had regular sleep-wake schedules (from 22:00$12623: 00 \mathrm{hr}$ to 06:00-07:00 hr). Travelling abroad, as well as use of alcohol and caffeine was 127 prohibited one week before and during the study. Coffee-drinkers were provided with 128 decaffeinated beverages. 
The blood samples were collected all throughout the year; in detail, 13 of the 17

130 perimenopausal women, and 10 of the 18 postmenopausal women were studied during winter 131 time (October to March). The participants spent one adaptation night (from 19:30 to 08:00 hr; 132 lights-off at 23:00, lights-on at 07:00 hr) in the sleep laboratory. In the following morning, a 133 blood sample was taken for baseline serum FSH and estradiol (E2) measurements. On the 134 following evening, the women returned to the laboratory at 19:30 hr for the baseline sleep 135 recording (lights-off at 23:00, lights-on at 07:00 hr), which was repeated also through the 136 third night. During the night only red light was allowed for illumination if needed. Therefore, 137 during the study period the participants spent their time inside a building, in a dark room 138 without windows, with strictly controlled nighttime illumination levels; this has limited the 139 possible influence of different photoperiods in different subjects. The study was performed by 140 similar timetable in all subjects and food was provided by the sleep laboratory.

141 On the evening before the third night an indwelling catheter was inserted into a forearm vein 142 to permit a 24-hour blood sampling at 20-minute intervals, starting from 21:00 hr. At night 143 (from 21:00 to 07:00 hr) the catheter was connected to a plastic tube extending into an 144 adjacent room: this allowed repeated blood sampling without disturbing the woman's sleep. 145 The catheter was kept patent with a slow heparinized saline infusion. Thus, melatonin 146 measurements were available for 20-minute interval samples between 21:00 and midnight, 147 and from 06:00 to 09:00 hr; measurements on one-hour interval samples were available 148 between midnight and 06:00 hr. All perimenopausal women were examined in the beginning 149 of their menstrual cycle (i.e., in the follicular phase).

150 The blood samples were drawn into EDTA tubes and placed in the refrigerator for $20 \mathrm{~min}$. 151 Thereafter, they were centrifuged to separate serum, which was frozen at $70^{\circ} \mathrm{C}$ until assayed. 152 The inter-assay coefficients of variation were $2.3 \%$ for FSH at a concentration of 44.8 IU/l 153 and $8.5 \%$ for $\mathrm{E} 2$ at a concentration of $0.18 \mathrm{nmol} / \mathrm{l}$, and the analytical sensitivities were 0.05 
IU/l and $0.05 \mathrm{nmol} / 1$ respectively. For melatonin analyses the serum samples were first extracted with chloroform and then assayed by radioimmunoassay with an iodinated melatonin tracer and a melatonin-specific antiserum. ${ }^{41}$ The lowest detectable concentration by the method was $1.3 \mathrm{pg} / \mathrm{ml}(5.7 \mathrm{pmol} / \mathrm{l})$, and the intra-assay and inter-assay coefficients of variation were from 6.7 to $9.5 \%$ and from 9.8 to $12.5 \%$, respectively. The following melatonin indicators were derived: 1 . the mean nighttime serum melatonin concentration from lights-off (at 23:00 hr) to lights-on (at 07:00 hr); 2. the range and mean of maximum and minimum levels of nighttime serum melatonin concentration (from lights-off to lights-on); 3. the nighttime melatonin exposure level: after the interpolation of melatonin exposure level curve, the area under melatonin exposure curve (AUC, from lights-off to 164 lights-on) was calculated for each individual, and the mean, quartile and median values of 165 melatonin exposure levels were calculated; 4. the duration of nighttime melatonin secretion: 166 the total amount of time (in hours) when serum melatonin levels (circulating melatonin) were $167 \geq 10 \mathrm{pg} / \mathrm{ml}$, where $10 \mathrm{pg} / \mathrm{ml}$ is the usual threshold for melatonin onset; ${ }^{42,43} 5$. the melatonin 168 peak time: the clock time of the peak of melatonin secretion; and 6. the time from lights off to 169 melatonin peak time (in hours).

Depressive symptoms during the past four weeks were evaluated with the Beck Depression 173 Inventory (BDI, a sum score, with the range of 0-63), ${ }^{44}$ and current anxiety level with the 174 State-Trait Anxiety Inventory (STAI, a sum score, with the range of 20-80). ${ }^{45}$ Insomnia (a 175 sum score, with the range of 5-25) and sleepiness (a sum score, with the range of 5-25) during the past three months were evaluated using the Basic Nordic Sleep Questionnaire (BNSQ), ${ }^{46}$ with lower score referring to better sleep (i.e., low levels of sleeping problems and sleepiness; see Appendix 1, Supplemental Digital Content 1, which reports the questions concerning 
179 insomnia and sleepiness of the BNSQ). In addition, the subjective sleep score (a sum score 180 with the range of 6-20) of the preceding blood-sampling night in the laboratory was assessed 181 in the morning by questions on sleep quality, sleep efficiency, sleep latency, number of 182 awakenings, too early morning awakening and morning tiredness, with lower number 183 referring to better sleep or to a low level of sleeping problems (see Appendix 2, Supplemental 184 Digital Content 2, which reports the questions concerning the sleep of preceding night used to 185 calculate the subjective sleep score). Climacteric vasomotor symptoms were scored with two 186 questions on the past six months (night sweats and hot flashes). The frequency of the symptoms (a sum score, with the range of 2-8) was determined on the following four-point scale: one ("seldom or never"), two ("approximately once a month"), three ("approximately once a week"), four (“almost every day"). The current quality of life (an index score, with the 190 range of from -0.011 to +1 ) was assessed using the EuroQoL quality of life questionnaire (EQ-5D) and the EQ-5D visual analogy scale (VAS, a scale score, with the range of 1-100). ${ }^{47}$ The EQ-5D index was calculated through a specific algorithm which considers a weight for each dimension. ${ }^{48}$ The questionnaires were administered during the day after the blood 194 sampling.

Statistical analysis

After testing for normality of the distribution (the Kolmogorov-Smirnov test), bivariate analyses were calculated to study the differences between perimenopausal and postmenopausal women using Student's $t$-test for comparison of the mean values. A $p$-value of $<0.05$ was considered significant. The mean, maximum and minimum levels of nighttime serum melatonin concentrations, the mean nighttime melatonin exposure level, the duration of nighttime melatonin secretion and the melatonin peak time in perimenopausal $v s$. postmenopausal women were compared by means of t-test and Wilcoxon rank-sum test. 
204 Bivariate Pearson correlation analyses were performed separately in perimenopausal women

205 and in postmenopausal women between nighttime serum melatonin concentrations, melatonin 206 exposure level, duration of nighttime melatonin secretion and the time from lights off to 207 melatonin peak, vs. independent variables including FSH, E2, body-mass index (BMI), BDI, 208 STAI, BNSQ insomnia, BNSQ sleepiness, subjective sleep score, climacteric vasomotor 209 symptom score and quality of life (EQ-5D index and EQ-5D VAS). Interaction analyses were 210 performed to test the associations between nighttime melatonin exposure (AUC) and, 211 alternatively, each of the independent variables that differed (or tended to differ) between 212 perimenopausal and postmenopausal women (i.e., FSH levels, E2 levels, BMI, BDI, BNSQ 213 insomnia and climacteric vasomotor scores). Menopausal status was entered in each model as 214 a controlling variable. The statistical analyses were performed using the SPSS/PASW 215 software version 18.0 (SPSS Inc., Chicago, IL, USA) and the R program. ${ }^{49}$

\section{Results}

218 The basic characteristics of the participants are described in Table 1. As determined,

219 perimenopausal women were younger and had lower FSH levels and higher E2 levels than 220 postmenopausal women. In addition, perimenopausal women had lower BMI and less 221 climacteric vasomotor symptoms. A tendency towards lower BDI and BNSQ insomnia scores 222 was found in perimenopausal women. No differences were found in respect to anxiety scores 223 on the STAI, sleepiness scores on the BNSQ or subjective sleep questionnaire, or the quality 224 of life on the EQ-5D index or EQ-5D VAS.

225 Data on melatonin levels was available for 17 perimenopausal and 17 postmenopausal women 226 (data missing from one postmenopausal woman). Values of melatonin indicators in 227 perimenopausal and postmenopausal women are reported in Table 2. Mean nighttime serum 228 melatonin concentrations, maximum and minimum levels, as well as mean nighttime 
melatonin exposure level (AUC) were lower in postmenopausal compared with

230 perimenopausal women (Table 2 and Figure 1). Although the melatonin peak time did not

231 differ between the two groups, the duration of the nighttime melatonin secretion (serum level

$232 \geq 10 \mathrm{pg} / \mathrm{ml} ; 43.1 \mathrm{pmol} / \mathrm{l})$ approached the significant level for a longer duration in

233 perimenopausal women than in postmenopausal women (6 h $47 \mathrm{~min}$ vs. $6 \mathrm{~h} 22 \mathrm{~min}$,

234 respectively; $\mathrm{p}=0.058$ ).

235 The mean nighttime melatonin concentration and age did not correlate. Further, mean

236 nighttime serum melatonin concentration did not correlate with FSH levels or E2 levels, BMI,

237 BDI, STAI, BNSQ insomnia, BNSQ sleepiness, subjective sleep of the preceding night,

238 climacteric vasomotor score or the quality of life (EQ-5D score and EQ-VAS) either in

239 perimenopausal or postmenopausal women. No correlations were found between nighttime

240 melatonin exposure level (AUC) and any of the independent variables either in

241 perimenopausal or postmenopausal women. In the perimenopausal group, the time from lights

242 off to melatonin peak correlated with scores on the STAI $(\mathrm{r}=0.55, p=0.022)$, i.e., the later the

243 melatonin peak, the higher the anxiety level. In addition, the duration of nighttime melatonin

244 secretion (serum levels $\geq 10 \mathrm{pg} / \mathrm{ml} ; 43.1 \mathrm{pmol} / \mathrm{l}$ ) correlated with the EQ5D-VAS scores

$245 \quad(\mathrm{r}=0.74 ; p<0.001)$, i.e. the longer the duration of nighttime melatonin secretion, the better the

246 quality of life. No correlations were found in the postmenopausal group.

247 In interaction analyses, no association was found between nighttime melatonin exposure level

248 (AUC) and FSH or E2 levels, BMI, BDI, BNSQ insomnia or climacteric vasomotor scores

249 after controlling for the menopausal status.

250

$251 \quad$ Discussion

252 From these results using serial blood draws, we are among the first to observe that as 253 compared to perimenopausal women, postmenopausal women had reduced nighttime 
melatonin concentrations at each time point. However, the nighttime pattern of rise and fall in melatonin levels, including the melatonin peak time was similar for both groups. Although not reaching statistical significance, the nighttime duration of melatonin secretion (the circulating melatonin concentrations equal to or more than $10 \mathrm{pg} / \mathrm{ml}$ ) was longer in the perimenopausal women. Postmenopausal women had more depressive, insomnia and climacteric vasomotor symptoms than perimenopausal women, but there was no evidence that these symptoms were related to the melatonin levels for either group.

Our findings of lower nighttime melatonin concentrations in postmenopausal women may be a reflection of the well-known age-related decrease of melatonin levels, ${ }^{16,17}$ where the transition into menopause may be itself considered as a dimension of aging, in specific of the hypothalamus-pituitary-ovarian system. In this respect, it is possible that the menopauserelated hormonal alterations, or the accompanying mood, sleep and vasomotor symptoms, may modulate melatonin activity, e.g. by accelerating its reduction. On the other hand, it is also possible that the age-related changes in melatonin, whether or not attributable to the hypothalamus-pituitary-ovarian-axis-related hormone changes, contribute themselves to the

271 To date, only a few works have focused on this issue. In the study of Okatani et al. ${ }^{20}$ the nighttime serum melatonin concentration (measured via 2-hour interval samples between 20.00 and 08:00 hr) was higher in the oldest premenopausal women (aged 46 to 50 years, i.e. likely perimenopausal) compared to younger premenopausal women with or without oophorectomy. Additionally, they found that nocturnal melatonin concentration and secretion decreased steeply in the first 15 years since the beginning of menopause, and continued to decrease more gradually thereafter. In that study reproductive state was determined on the basis of menstrual records. Vakkuri et al. ${ }^{19}$ studied 77 women aged 30 to 75 years, dividing 
them into premenopausal and postmenopausal groups on the basis of their menstrual records, and thereafter into six further age groups. Nocturnal (20.00-08.00 hr) urinary excretion of melatonin and morning (09.00 hr) serum melatonin were measured. Urinary melatonin levels were found to decline during the menopausal transition, the most significantly in women aged 40-44 years, followed by those over 50 years; also, the serum morning melatonin levels tended to be lower in women aged 60 years and over compared to women younger than 40 years. Keeping in mind the different age ranges of the participants, as well as the different melatonin sampling (nocturnal urinary and morning serum samples in the Vakkuri et al. study, ${ }^{19}$ vs. serial nocturnal serum samples in our current work), these results are in line with our findings of decreased melatonin concentration in postmenopause. Since the drop in melatonin levels was most notable far before menopausal age, the authors concluded that it could be permissively linked to the initiation of menopause. Frequently, changes in the hypothalamus-pituitary-ovarian function start several years before the actual cessation of menstrual periods, i.e. at the age where the drop in melatonin level was more evident. Further, Vakkuri et al. ${ }^{19}$ reported a negative correlation between urinary melatonin and serum FSH.

294 Fernandez et al. ${ }^{18}$ found the lowest values of morning serum (but not urinary) melatonin 295 levels in postmenopausal women. Moreover, they found no correlation between melatonin and $\mathrm{FSH}, \mathrm{E} 2$ or progesterone levels during the perimenopausal period, but a negative correlation between FSH and melatonin levels in the postmenopausal women. We did not find any association between melatonin and FSH or E2 levels in either perimenopausal or postmenopausal women. A plausible reason for the lack of such correlation was a 301 not be ignored that we used the mean value of repeated nocturnal serum samples, instead of an overnight urinary sample as in the study of Vakkuri et al. ${ }^{19}$ or a single morning serum sample as in the study of Fernandez et al. ${ }^{18}$ Even though urinary melatonin (or its metabolite) 
304 sampling technique is among the most practical ones for the assessment of melatonin

305 secretion, given its limited possibility of repeated samples, it may lack in precision. Saliva

306 sampling is also a practical and reliable technique, which allows repeated samples; however,

307 it can hardly be used for overnight assessment. On the contrary, repeated blood samples can

308 be more easily taken at frequent intervals during night without or with limited sleep

309 disruption. In addition, the levels of melatonin are higher in the plasma than in the saliva, thus

310 implying a better resolution and sensitivity. In detail, overnight blood sampling at frequent

311 intervals seems to be the most informative technique for the assessment of melatonin

312 profile. ${ }^{50}$

313 Our results also suggest that the nighttime pattern of rise and fall in melatonin levels,

314 including the melatonin peak time, does not significantly differ between perimenopausal and

315 postmenopausal women. This finding is in contrast with the earlier report of Walters et al. ${ }^{51}$

316 on the advanced phase of melatonin secretion in postmenopausal compared with

317 premenopausal women during one-night sleep deprivation. However, even though in the same

318 study the melatonin onset was found to precede the onset of subjective sleepiness equally in

319 the premenopausal and postmenopausal women, the time between the melatonin onset and

320 that of sleepiness was longer in postmenopausal than premenopausal women. ${ }^{51}$ Possible

321 explanations for these different outcomes may be the different study designs, since Walters et

322 al. ${ }^{51}$ assessed salivary melatonin from samples collected hourly for 22 hours during sleep

323 deprivation under constant routine conditions, and studied younger groups (premenopausal

324 aged 38-46 years, postmenopausal aged 53-57 years), whereas we used repeated serum

325 samples and older study groups (perimenopausal aged 43-51 years, postmenopausal aged 58-

32671 years) in normal sleeping condition.

327 Melatonin concentration and secretion pattern seemed not to be related to climacteric

328 vasomotor symptoms or to BMI either in perimenopausal or in postmenopausal women. As 
expected, postmenopausal women had more climacteric vasomotor symptoms than

330 perimenopausal women. They also had a higher BMI, which is known to increase with the 331 transition to menopause, even with small differences according to the type of menopause. ${ }^{52}$ It 332 is possible that, besides other known factors such as changes in estrogen and FSH levels, the 333 menopause- and age-related changes in melatonin levels also contribute to these symptoms 334 and body changes in postmenopausal women. A recent animal study showed that 335 administration of melatonin was more effective than estrogen therapy in reversing the 336 glycemic and lipid dysregulation as well as in restoring the increased BMI after the 337 ovariectomy. ${ }^{53}$ According to another study administration of melatonin to perimenopausal and 338 postmenopausal women led to a tendency of reduction in climacteric symptoms. ${ }^{54}$ However, 339 other studies did not support these results. ${ }^{55,56}$ Our correlation and interaction analyses did not 340 find any association between melatonin and climacteric symptoms or BMI, even after $341 \quad$ controlling for the effect of menopausal status.

342 Also, melatonin concentration and secretion pattern seemed not to be related to depressive 343 symptoms or sleep disturbances either in perimenopausal or in postmenopausal women. In our 344 sample, postmenopausal women tended to have more depressive and insomnia symptoms.

345 Instead, anxiety and the quality of life scores did not differ between perimenopausal and 346 postmenopausal women. This is in line with the well-known increased risk of mood 347 symptoms and disorders ${ }^{22}$ and decrease in sleep quality ${ }^{57-60}$ during the menopausal transition. 348 In early $\left(<5\right.$ years) versus late $(>5$ years $)$ postmenopausal women, Hachul et al. ${ }^{61}$ found more 349 depression, anxiety and sleepiness among the latter group. In general, it has been reported that 350 perimenopausal and especially postmenopausal women suffer from subjective sleep problems 351 more than premenopausal women. ${ }^{57-60}$ In this respect, a prospective study ${ }^{62}$ showed that even 352 after controlling for age and other confounding factors, women had higher odds for both 353 moderate and severe self-reported sleep problems when transiting from premenopause to 
perimenopause, and even higher odds when transiting to postmenopause. However, perhaps because of the small sample size, correlation and interaction analyses did not detect any significant associations between melatonin and BDI or BNSQ insomnia scores. Hence, these findings do not support our original hypothesis that low melatonin levels associate with depressive, anxiety or sleep symptoms in postmenopausal women. It is of note that melatonin levels was associated with other parameters in the perimenopausal group: a delayed peak time 360 was associated with a higher level of anxiety, while a longer duration of melatonin secretion 361 was associated with better quality of life. It is possible that higher levels of anxiety postponed 362 the onset of sleep and subsequently the peak of melatonin secretion, whereas longer durations 363 of melatonin secretion could improve sleep quality and subsequently the quality of life.

364 This study was the first one to use repeated serum sampling technique to evaluate the 365 interrelationship of melatonin secretion and menopausal status. This seems to be the best 366 technique as assessment of melatonin phase, duration and amplitude are concerned, in particular when frequent (20-30 minutes) samples are provided.$^{50}$ Even if the study was not 368 carried out under constant routine conditions, which would have been the most appropriate 369 technique, nevertheless the high-frequency collection of serum samples under strictly controlled sleep laboratory conditions ensured the good quality of the samples in order to monitor the pattern of melatonin concentrations. In addition, several confounding factors were effectively ruled out by the accurate exclusion criteria, such as irregular sleep-wake 373 schedules, the use of HT and other medications, smoking as well as the use of alcohol or 374 drugs. The main limitation of our study is a rather small sample size in the context of a convenience sampling design. This, along with the elevated inter-individual variability in melatonin levels, may partly explain the absence of any significant correlation between melatonin and FSH or E2 levels, or most of the mood and sleep symptoms. However, the sample size was 
comparable with that of other studies in the field, and even with this limited sample size,

380 significant differences in melatonin levels were detected. The study was carried out on a 381 healthy population, preventing the generalization of the results to populations with common 382 diseases. Further, some of the women in the perimenopausal group had regular menstruation, 383 categorizing them as premenopausal. In addition, it must be noticed that mood, sleep and 384 climacteric symptoms and quality of life were retrospectively assessed with self-reported 385 questionnaires that covered different timeframes of recall (ranging from current quality of life 386 to climacteric symptoms in the past six months), being a potential weakness in the 387 measurement of self-reported data. However, the reliability and validity of most of these 388 instruments have been tested and found to be reliable. ${ }^{46,63,64}$ The melatonin sampling took 389 place throughout the year, likely influencing the results. However, during the visit in the sleep 390 laboratory the participants spent their time inside the building and the nighttime illumination 391 levels were strictly controlled. Finally, the cross-sectional design of the study did not allow 392 any causal conclusions.

Conclusions

395 Our results confirm lower nighttime serum melatonin concentrations and exposure levels in 396 postmenopausal women compared with perimenopausal women, with no difference in 397 melatonin peak time. There was also a tendency towards longer duration of melatonin 398 secretion in perimenopausal women. Further research with prospective follow-up studies 399 during menopausal transition is needed to better understand the nature of these differences. 400 Changes in melatonin levels were not related to mood, sleep quality, vasomotor symptoms or 401 quality of life either in perimenopause or postmenopause. This finding needs to be confirmed 402 in larger studies. Whether the beneficial effect of HT on alleviation of these symptoms in 403 menopause is partly regulated via melatonin needs to be verified. 
$405 \quad$ References

406 1. Bubenik GA. Gastrointestinal melatonin. Localization, function and clinical relevance. Dig 407 Dis Sci 2002;47:2336-2348.

408 2. Arendt J. Melatonin: characteristics, concerns, and prospects. J Biol Rhythms 2005;20:291409303.

$4103 . \quad$ Okatani Y, Morioka N, Hayashi K. Changes in nocturnal pineal melatonin synthesis during 411 the perimenopausal period: relation to estrogen levels in female rats. J Pineal Res 1999;27:6541272

413 4. San Martin M, Touitou Y. Progesterone inhibits, on a circadian basis, the release of 414 melatonin by rat pineal perifusion. Steroids 2000;65:206-209.

415 5. Hernández-Díaz FJ, Sánchez JJ, Abreu P, et al. Estrogen modulates alpha 1 /beta416 adrenoceptor-induced signaling and melatonin production in female rat pinealocytes. 417 Neuroendocrinology 2001;73:111-122.

418 6. Caufriez A, Leproult R, L’Hermite-Baleriaux M, Kerkhofs M, Copinschi G. Progesterone 419 prevents sleep disturbances and modulates GH, TSH, and melatonin secretion in postmenopausal $420 \quad$ women. J Clin Endocrinol Metab 2011;96:E614-E623.

421 7. Okatani Y, Sagara Y. Amplification of nocturnal melatonin secretion in women with 422 functional secondary amenorrhoea: relation to endogenous oestrogen concentration. Clin $423 \quad$ Endocrinol 1994;41:763-770.

424 8. Ishizuka B, Fusama S, Hirai K, et al. Melatonin secretion from organ-cultured pineal glands 425 of rats: modulation by gonadectomy and gonadotropin-releasing hormone agonist administration. Eur J Endocrinol 2000;142:387-392. 
9. Kostoglou-Athanassiou I, Athanassiou P, Treacher DF, Wheeler MJ, Forsling ML.

428 Neurohypophysial hormone and melatonin secretion over the natural and suppressed menstrual $429 \quad$ cycle in premenopausal women. Clin Endocrinol 1998;49:209-216.

430 10. Woo MMM, Tai C-J, Kang SK, Nathwani PS, Pang SF, Leung PCK. Direct action of 431 melatonin in human granunlosa-luteal cells. J Clin Endocrinol Metab 2001;86:4789-4797.

432 11. Cos S, González A, Martínez-Campa C, Mediavilla MD, Alonso-González C, Sánchez433 Barceló EJ. Melatonin as a selective estrogen enzyme modulator. Curr Cancer Drug Target $434 \quad 2008 ; 8: 691-702$.

435 12. Voordouw BC, Euser R, Verdonk RE, et al. Melatonin and melatonin-progestin 436 combinations alter pituitary-ovarian function in women and can inhibit ovulation. $J$ Clin $437 \quad$ Endocrinol Metab 1992;74:108-117.

438 13. Reiter RJ. Melatonin and human reproduction. Ann Med 1998;30:103-108.

439 14. Brzezinski A, Lynch HJ, Siebel MM, Deng MH, Nader TM, Wurtman RJ. The circadian 440 rhythm of plasma melatonin during the normal menstrual cycle and in amenorrheic women. $J$ $441 \quad$ Clin Endocrinol Metab 1988;66:891-895.

442 15. Luboshitzky R, Lavi S, Thuma I, Lavie P. Increased nocturnal melatonin secretion in male 443 patients with hypogonadotropic hypogonadism and delayed puberty. J Clin Endocrinol Metab $444 \quad 1995 ; 80: 2144-2148$.

445 16. Iguchi H, Kato K, Ibayashi H. Age-dependent reduction in serum melatonin concentrations 446 in healthy human subjects. J Clin Endocrinol Metabol 1982;55:27-29.

447 17. Kennaway DJ, Lushington K, Dawson D, Lack L, Van den Heuvel C, Rogers N. Urinary 6448 sulfatoxymelatonin excretion and aging: new results and a critical review of the literature. $J$ $449 \quad$ Pineal Res 1999;27:210-220. 
18. Fernandez B, Malde JL, Montero A, Acuña D. Relationship between adenohypophyseal and

451 steroid hormones and variations in serum and urinary melatonin levels during the ovarian cycle,

452 perimenopause and menopause in healthy women. J Steroid Biochem 1990;35:257-262.

453 19. Vakkuri O, Kivelä A, Leppäluoto J, Valtonen M, Kauppila A. Decrease in melatonin

454 precedes follicle-stimulating hormone increase during perimenopause. Eur J Endocrinol

$455 \quad$ 1996;135:188-192.

456 20. Okatani Y, Morioka N, Wakatsuki A. Changes in nocturnal melatonin secretion in 457 perimenoausal women: correlation with endogenous estrogen concentrations. J Pineal Res $458 \quad 2000 ; 28: 111-118$.

459 21. Kotlarczyk MP, Lassila HC, O’Neil CK, et al. Melatonin osteoporosis prevention study 460 (MOPS): a randomized, double-blind, pacebo-controlled study examining the effects of 461 melatonin on bone health and quality of life in perimenopausal women. $J$ Pineal Res $462 \quad 2012 ; 52: 414-426$.

463 22. Kessler RC, McGonagle KA, Swartz M, Blazer DG, Nelson CB. Sex and depression in the 464 national comorbidity survey I: lifetime prevalence, chronicity and recurrence. J Affect Disord $465 \quad$ 1993;29:85-96.

466 23. Freeman EW, Sammel MD, Lin H, Nelson DB. Associations of hormones and menopausal 467 status with depressed mood in women with no history of depression. Arch Gen Psychiatry $468 \quad 2006 ; 63: 375-382$.

469 24. Deecher D, Andree TH, Sloan D, Schechter LE. From menarche to menopause: Exploring 470 the underlying biology of depression in women experiencing hormonal changes.

$471 \quad$ Psychoneuroendocrinology 2008;33:3-17.

472 25. Nowakowski S, Meliska CJ, Martinez LF, Parry BL. Sleep and menopause. Curr Neurol $473 \quad$ Neurosci Rep 2009;9:165-172. 
26. Hachul H, Andersen ML, Bittencourt LR, Santos-Silva R, Conway SG, Tufik S. Does the reproductive cycle influence sleep patterns in women with sleep complaints? Climacteric 2010;13:594-603.

27. Polo-Kantola P. Sleep problems in midlife and beyond. Maturitas 2011;68:224-232.

28. Eichling PS, Sahni J. Menopause related sleep disorders. J Clin Sleep Med 2005;1:291-300.

29. Polo-Kantola P. Sleep and menopause. Womens Health 2007;3:99-106.

30. Beck-Friis J, von Rosen D, Kjellman BF, Ljunggren JG, Wetterberg L. Melatonin in relation to body measures, sex, age, season and the use of drugs in patients with major affective disorders and healthy subjects. Psychoneuroendocrinology 1984;9:261-277.

31. Claustrat B, Chazot G, Brun J, Jordan D, Sassolas G. A chronobiological study of melatonin and cortisol secretion in depressed subjects: plasma melatonin, a biochemical marker in major depression. Biol Psychiatr 1984;19:1215-1228.

32. Beck-Friis J, Kjellman BF, Aperia B, et al. Serum melatonin in relation to clinical variables in patients with major depressive disorder and a hypothesis of a low melatonin syndrome. Acta Psychiatr Scand 1985;71:319-330.

33. Brown R, Kocsis JH, Caroff S, et al. Differences in nocturnal melatonin secretion between melancholic depressed patients and control subjects. Am J Psychiatry 1985;142:811-816. 34. Crasson M, Kjiri S, Colin A, et al. Serum melatonin and urinary 6-sulfatoxymelatonin in major depression. Psychoneuroendocrinology 2004;29:1-12. menopausal depression: role of years post-menopause, follicle-stimulating hormone, sleep end time, and Body Mass Index. J Clin Endocrinol Metab 2008;93:54-60. 
498 benzodiazepines on a conflict procedure. Prog Neuro-Pscyhopharmacol \& Biol Psychiatr 2000;24:117-129.

500 37. Papp M, Litwa E, Gruca P, Mocaer E. Anxiolytic-like activity of agomelatine and 501 melatonin in three animal models of anxiety. Behav Pharmacol 2006;17:9-18.

502 38. Stein DJ, Ahokas AA, De Bodinat C. Efficacy of agomelatine in generalized anxiety 503 disorder. A randomized, double-blind, placebo-controlled study. J Clin Psychopharmacol $504 \quad 2008 ; 28: 561-566$.

505 39. Cardinali DP, Srinivasan V, Brzezinski A, Brown GM. Melatonin and its analogs in $506 \quad$ insomnia and depression. J Pineal Res 2012;52:365-375.

507 40. Kalleinen N, Polo-Kantola P, Himanen S-L, et al. Sleep and the menopause - do 508 postmenopausal women experience worse sleep than premenopausal women? Menopause Int $509 \quad 2008 ; 14: 97-104$.

510 41. Vakkuri O, Leppäluoto J, Vuolteenaho O. Development and validation of a melatonin 511 radioimmunoassay using radioiodinated melatonin as tracer. Acta Endocrinol 1984;106:152-157. 512 42. Lewy AJ. The dim light melatonin onset, melatonin assays and biological rhythms research 513 in humans. Biol Signals Recept 1999; 8:79-83.

514 43. Lewy AJ, Culter NL, Sack RL. The endogenous melatonin profile as a marker for circadian $515 \quad$ phase position. J Biol Rhythms 1999; 14:227-236.

516 44. Beck AT, Ward CH, Mendelson M, Mock J, Erbaugh J. An inventory for measuring 517 depression. Arch Gen Psych 1961;4:561-571.

518 45. Spielberger CD, Gorssuch RL, Lushene PR, Vagg PR, Jacobs GA. Manual for the State$519 \quad$ Trait Anxiety Inventory. Consulting Psychologists Press, Inc. 1983.

520 46. Partinen M, Gislason T. Basic Nordic Sleep Questionnaire (BNSQ): a quantitated measure 521 of subjective sleep complaints. J Sleep Res 1995;4:150-155. 
523 population as measured by EuroQol. EuroQol, Plenary meeting: Barcelona, Spain, Oct 3-5, 524 1995: Discussion papers. Barcelona: Catalan Institute of Public Health; 1996; p. 161-172.

525 48. www.euroqol.org/about-eq-5d/publications/user-guide.html.

526 49. Team R Development Core. R: A Language and Environment for Statistical Computing.

527 Vienna, Austria, 2011. http://www.R-project.org/

528 50. Benloucif S, Burgess HJ, Klerman EB, et al. Measuring melatonin in humans. J Clin Sleep $529 \quad$ Med 2008;4:66-69.

530 51. Walters JF, Hampton SM, Ferns GA, Skene DJ. Effect of menopause on melatonin and 531 alertness rhythms investigated in constant routine conditions. Chronobiol Int 2005;22:859-872.

532 52. Gibson CJ, Thurston RC, El Khoudary SR, Sutton-Tyrrell K, Matthews KA. Body mass 533 index following natural menopause and hysterectomy with and without bilateral oophorectomy. $534 \quad$ Int J Obes 2013;37:809-813.

535 53. Baxi D, Singh PK, Vachhrajani K, Ramachandran AV. Melatonin supplementation therapy 536 as a potent alternative to ERT in ovariectomized rats. Climacteric 2012;15:382-392.

537 54. Bellipanni G, Di Marzo F, Blasi F, Di Marzo A. Effects of melatonin in perimenopausal and 538 menopausal women. Our personal experience. Ann NY Acad Sci 2005;1057:393-402.

539 55. Secreto G, Chiechi LM, Amadori A, et al. Soy isoflavones and melatonin for the relief of 540 climacteric symptoms: a multicenter, double-blind, randomized study. Maturitas 2004;47:11-20.

541 56. Kripke DF, Kline LE, Shadan F, Dawson A, Poceta JS, Elliott JA. Melatonin effects on 542 luteinizing hormone in postmenopausal women: a pilot clinical trial NCT00288262. BMC $543 \quad$ Womens Health 2006;6:8.

544 57. Kuh DL, Wadsworth M, Hardy R. Women's health in midlife: the influence of the 545 menopause, social factors and health in earlier life. Br J Obstet Gynaecol 1997;104:923-933. 
546 58. Owens JF, Matthews KA. Sleep disturbance in healthy middle-aged women. Maturitas $547 \quad 1998 ; 30: 41-50$.

548 59. Kravitz HM, Ganz PA, Bromberger J, Powell LH, Sutton-Tyrrell K, Meyer PM. Sleep 549 difficulty in women at midlife. Menopause 2003;10:19-28.

550 60. Berecki-Gisolf J, Begum N, Dobson AJ. Symptoms reported by women in midlife:

551 menopausal transition or aging? Menopause 2009;16:1021-1029.

552 61. Hachul H, Bittencourt LRA, Soares JM Jr, Tufik S, Baracat EC. Sleep in post-menopausal 553 women: differences between early and late post-menopause. Eur J Obstet Gynecol Reprod Biol $554 \quad 2009 ; 145: 81-84$.

555 62. Tom SE, Kuh D, Guralnik JM, Mishra GD. Self-reported sleep difficulty during the 556 menopausal transition: results from a prospective cohort study. Menopause 2010;17:1128$557 \quad 1135$.

558 63. Beck AT, Steer RA, Carbin MG. Psychometric properties of the Beck Depression Inventory: 559 Twenty-five years of evaluation. Clinical Psychology Review 1988;8:77-100.

560 64. Spielberger CD. State-Trait Anxiety Inventory: Bibliography (2nd ed.). Consulting $561 \quad$ Psychologists Press, Palo Alto, CA, 1989. 
563 Figure 1. Mean nighttime serum melatonin concentrations (pg/ml) of perimenopausal and 564 postmenopausal women.

565 
- Supplemental Digital Content 1. Appendix that reports the BNSQ questions concerning insomnia and sleepiness

- Supplemental Digital Content 2. Appendix that reports the questions concerning the sleep of preceding night used to calculate the subjective sleep score 